\title{
Limited mechanical effects of intermuscular myofascial connections within the intact rat anterior crural compartment
}

\author{
Chris Tijs, Jaap H. van Dieën, Huub Maas* \\ Department of Human Movement Sciences, Faculty of Behaviour and Movement Sciences, Vrije Universiteit Amsterdam, MOVE Research Institute Amsterdam, \\ The Netherlands
}

\section{A R T I C L E I N F O}

\section{Article history:}

Accepted 8 July 2016

\section{Keywords:}

Tibialis anterior

Extensor digitorum longus

3D kinetics

Co-activation

Mechanical interaction

\begin{abstract}
A B S T R A C T
Skeletal muscles of the rat anterior crural compartment are mechanically connected by epimuscular myofascial connections, but the relevance for mechanical muscle function within physiological ranges of joint motion is unclear. We evaluated the net effect at the ankle joint of epimuscular myofascial connections between tibialis anterior (TA) and extensor digitorum longus (EDL) muscles in the rat $(n=8)$ and determined which anatomical structures may mediate such epimuscular mechanical interactions. We assessed (1) effects of knee angle (i.e. changes in EDL length and position relative to TA) and interactions of knee angle with fasciotomy and proximal EDL tenotomy on TA ankle moment and (2) the effect of knee angle on TA and EDL ankle moment summation. Knee angle was varied between $60^{\circ}$ and $130^{\circ}$. Ankle angle was kept constant $\left(90^{\circ}\right)$. TA and EDL were excited individually and simultaneously (TA\&EDL). The mathematical sum of individual TA and EDL moments was compared with the moment exerted by TA\&EDL to assess the extent of non-additive ankle moment summation. Magnitude of TA ankle moment was not affected by knee angle, but frontal plane moment direction was. However, dissections indicated that this was not caused by the compartmental fascia or EDL length changes. Moment summation was non-additive in magnitude $(+1.1 \pm 1.1 \%$ mean \pm s.d. $)$ and frontal plane direction. The latter was affected by knee angle and ranged from $+0.2 \pm 0.3^{\circ}$ at $60^{\circ}$ to $+1.1 \pm 0.6^{\circ}$ at $130^{\circ}$. As the net effects found were very limited, we conclude that myofascial connections between muscles in the anterior crural compartment have limited mechanical relevance during normal movement.
\end{abstract}

(c) 2016 Elsevier Ltd. All rights reserved.

\section{Introduction}

The myotendinous junction is the primary pathway for force transmission from the muscle via the tendon to the skeleton. Myofascial connections between a muscle's epimysium and surrounding structures can also transmit force (Huijing, 2009; Maas and Sandercock, 2010). Several studies, in which muscles within the rat anterior crural compartment were excited maximally and their tendons were severed from the skeleton, have shown that forces exerted at the tendon of a muscle kept at a constant muscletendon unit (MTU) length were dependent on the neighboring muscle's length (Huijing and Baan, 2008; Huijing et al., 2007; Maas et al., 2005; Meijer et al., 2006; Rijkelijkhuizen et al., 2007) or relative position (Maas et al., 2004). However, several of these imposed changes were beyond those found during normal

\footnotetext{
*Correspondence to: van der Boechorststraat 9, 1081 BT Amsterdam, The Netherlands.

E-mail address: h.maas@vu.nl (H. Maas).
}

movement. Therefore, the relevance for the mechanical function for physiological ranges of joint motion remains unclear.

Several imaging studies have reported effects of knee extension (i.e. proximal lengthening of gastrocnemius muscle) on deformations within passive soleus muscle in humans (Bojsen-Møller et al., 2010; Diong and Herbert, 2015; Huijing et al., 2011; Tian et al., 2012; Yaman et al., 2013), but others did not found changes in fascicle length (Kawakami et al., 1998). Although tissue deformations indicate effects of myofascial force transmission, the applied imaging techniques cannot provide a direct measure of the mechanical effects at the tendon or joint. A study that did assess the mechanical effect at the tendon reported sizable epimuscular myofascial force transmission between active rat ankle plantarflexor muscles for a physiological range of muscle lengths and relative muscle positions (Bernabei et al., 2015). In contrast, ankle moments exerted by soleus muscle in rats (Tijs et al., 2015a) and cats (Maas and Sandercock, 2008) were not affected by length changes of passive synergists, as imposed by changes in knee angle. These contradicting results suggest that epimuscular myofascial force transmission between soleus and gastrocnemius 
muscles may not be reflected in their moment vectors, as these muscles have largely comparable lines of action (Tijs et al., 2014).

For muscles within the anterior crural compartment, it is not clear if myofascial force transmission is present for physiological ranges of joint motion and if it is reflected in their moment vectors. These muscles have more distinct line of actions than the triceps surae muscles: while the mono-articular tibialis anterior (TA) exerts an ankle inversion moment, the poly-articular extensor digitorum longus (EDL) exerts an ankle eversion moment. Hence, the net effect of epimuscular myofascial force transmission at the ankle joint may be more pronounced.

The main purpose of this study was to evaluate the mechanical relevance of epimuscular myofascial connections between TA and EDL muscles in the rat for physiological ranges of joint motion. A secondary purpose was to assess which anatomical structures mediate such epimuscular mechanical effects.

\section{Materials and methods}

\subsection{Animals}

Data were obtained from 8 male Wistar rats (body mass: $313.4 \pm 10.5 \mathrm{~g}$, mean \pm s.d.). All procedures were in agreement with the guidelines and regulations concerning animal welfare and experimentation set forth by Dutch law, and approved by the Committee on Ethics of Animal Experimentation at the Vrije Universiteit Amsterdam (Permit Number: FBW 11-02).

According to standard procedures in our laboratory (Maas et al., 2001), intraperitoneally injected urethane was used to deeply anesthetize the animals, such that reflexes were fully suppressed. To maintain a core temperature of approximately $37{ }^{\circ} \mathrm{C}$, rats were placed on an electrical heating pad. Saline solution was applied frequently to prevent dehydration of exposed tissues. At the end of the experiment, animals were euthanized with an overdose of intracardially-injected pentobarbital sodium followed by a double-sided pneumothorax.

\subsection{Surgery}

Skin and biceps femoris muscle of the left hindlimb were removed. Medial and lateral malleoli and origin of medial and lateral collateral ligaments were marked and used as axis of rotation of ankle and knee joints, respectively. The femur was partly exposed to allow attachment of a metal clamp. The peroneal nerve was dissected free for placement of a bipolar cuff electrode. The superficial peroneal nerve and all branches of the deep peroneal nerve distally to the cuff electrode were cut, except one of two branches innervating TA muscle (Fig. 1). Because the EDL nerve branches were cut, EDL was excited via bipolar intramuscular wire electrodes that were inserted near the motor endplate located in the distal region. Therefore, only one EDL muscle compartment was most likely excited. Because EDL muscle belly is accessible only after a full longitudinal fasciotomy of the anterior crural compartment (Huijing et al., 2003; Maas et al., 2005), this was performed after the first set of measurements (see experimental protocol below).

\subsection{Fixation in experimental apparatus}

The left hindlimb was secured to the experimental set-up (for photographic overview see Tijs et al., 2014) by clamping the femur and attaching the foot to a 6 degrees-of-freedom load cell (Mini40-E, ATI, Apex, NC, USA). As EDL tendons insert onto the distal phalanx of digits II-V, the digits were secured at a $180^{\circ}$ angle for the metatarsophalangeal and interphalangeal joints. Ankle and knee joints were aligned with the set-up's rotational axes and their position relative to the origin of the load cell was measured using rulers integrated in the set-up.

\subsection{Experimental protocol}

TA muscle was excited by supramaximal stimulation of the common peroneal nerve (amplitude: $0.3-0.4 \mathrm{~mA}$, frequency: $100 \mathrm{~Hz}$, pulse width: $100 \mu \mathrm{s}$ ) via the cuff electrode connected to a constant current source (Digitimer DS3, Digitimer Ltd., Hertfordshire, England). Note that only the proximal TA nerve branch was excited. Based on existing data (Huijing and Baan, 2001a; Johnson et al., 2008; Maas et al., 2001) estimated maximum TA ankle moment was $21 \mathrm{mNm}$. Combining this with our data (TA moment $18.7 \pm 3.4 \mathrm{mNm}$; see Section 3 ) indicates that we excited $\sim 90 \%$ of TA muscle fibers. EDL muscle was excited via the intramuscular electrodes (amplitude: $0.7-2.0 \mathrm{~mA}$, frequency: $100 \mathrm{~Hz}$, pulse width: $100 \mu \mathrm{s}$ ). A pilot experiment revealed that $\sim 60 \%$ of maximum ankle moment exerted by EDL muscles

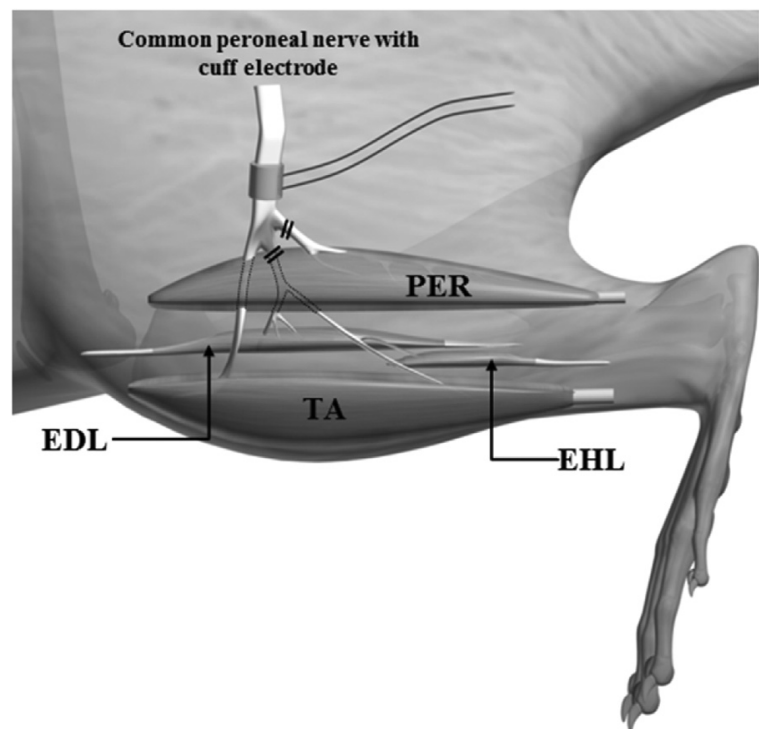

Fig. 1. Schematic view of branches of the common peroneal nerve. To excite TA muscle, a cuff electrode was placed around the common peroneal nerve proximally to the bifurcation into the deep peroneal nerve (to TA, EDL, EHL) and branches of the superficial peroneal nerve (to PER). Nerve branches innervating PER, EDL, EHL and distal TA were transected (solid black lines), leaving only the branch entering TA proximally intact. PER: peroneus. EDL: extensor digitorum longus. TA: tibialis anterior. EHL: extensor hallucis longus. For clarity, nerves innervating TA and EHL are displayed on top of the EDL muscle.

fibers was exerted when stimulating intramuscularly compared to supramaximal nerve stimulation.

We rotated the knee in the sagittal plane from $60^{\circ}$ to $130^{\circ}$ or vice versa (alternated between experiments) in steps of $10^{\circ}$, while sagittal plane ankle angle was kept constant at $90^{\circ}$. Such changes in knee angle were applied in two experimental conditions: (1) TA was activated while EDL remained passive. Effects of knee angle on magnitude and direction of TA ankle moment were assessed; (2) Both TA and EDL were activated. Differences between ankle moment exerted during simultaneous excitation of TA and EDL (TA\&EDL) and the sum of joint moments exerted by each muscle individually were assessed. This difference in joint moments is referred to as non-additive summation. If epimuscular myofascial connections between these muscles are of mechanical significance, such nonadditive summation will be knee angle dependent.

Knee angle was varied to change EDL length and position relative to TA. Ankle angle was kept constant to keep TA at a constant MTU length. Note that rat EDL spans the knee joint (Greene, 1935; Hildebrand et al., 1991). As knee extension decreases EDL MTU length (by $\sim 3 \mathrm{~mm}$ with changing knee angle from $45^{\circ}$ to $135^{\circ}$ (Maas et al., 2004)), the stiffness of epimuscular myofascial connections between TA and EDL may change, which was expected to affect the extent of intermuscular mechanical interaction. For reference, optimum MTU length of EDL is $\sim 50 \mathrm{~mm}$ and the length of the ascending limb of the MTU length-force curve is $\sim 10 \mathrm{~mm}$ (Huijing and Baan, 2003).

Similar to the imposed range of knee angles, previous studies found sagittal plane knee angles between $55^{\circ}$ and $125^{\circ}$ during rat locomotion (Bauman and Chang, 2010; Gruner et al., 1980). Ankle angles around $90^{\circ}$ occur especially during the late stance phase of level walking (Bauman and Chang, 2010), but are also found during upslope walking and swimming (Gruner and Altman, 1980; Schmidt and Fischer, 2011). Because the extent of EDL displacement relative to TA was considered highest in the sagittal plane, knee joint angle was changed only in this plane. For both joints, the angles in the other two planes were kept at $0^{\circ}$. Positioning the knee at the intended angle was done with maximal compliance to allow optimal alignment of the rotation axis of the set-up with the knee joint axis. After the knee was set at the intended angle, the component of the reaction force in the direction of the tibia was increased to a constant value (approximately $5 \mathrm{~N}$ ) to ensure similar compression of the lower hindlimb and minimal compliance in the ankle and knee joints.

Before data collection, history effects (e.g. effects of viscoelastic properties of muscles and connective tissues) were removed by TA and EDL excitation at the lowest and highest joint angles (i.e. MTU lengths) that we applied during the experiment (Huijing and Baan, 2001a). During the experiment, surgical interventions were performed resulting in three stages of dissection: (I) intact compartment; (II) after full longitudinal fasciotomy of the anterior crural compartment without disrupting myofascial connections between TA and EDL muscle bellies. The neurovascular tract that supplies TA was not damaged as fasciotomy was performed superficially and laterally, while the neurovascular tract is located deep and medially (Maas et al., 2001); (III) after proximal EDL tenotomy. For stage I, TA 
muscle was excited exclusively $(300 \mathrm{~ms})$. For stage II, two stimulation protocols were performed subsequently for each knee angle, which is based on a protocol that is explained in more detail elsewhere (Tijs et al., 2014). First, TA and EDL muscles were stimulated separately (TA: $300 \mathrm{~ms}$, EDL: $400 \mathrm{~ms}$ ). Second, EDL and TA were both stimulated but with tetanic trains of different lengths: EDL was stimulated for $400 \mathrm{~ms}$, while, after $200 \mathrm{~ms}$ of EDL stimulation, TA was stimulated for $200 \mathrm{~ms}$. The different trains were used to exclude possible effects of initial differences in EDL muscle excitation, which would bias our calculation of non-additive summation. The order of the stimulation protocols was alternated between experiments. For stage III, TA and EDL were excited separately (TA: $300 \mathrm{~ms}$, EDL: $400 \mathrm{~ms}$ ) at each knee angle to assess effects of proximal EDL tenotomy on isometric TA and EDL ankle moments. Two minutes rest periods were allowed between stimulation protocols. Video recordings (PANASONIC HC-V720, $1920 \times 1080$ pixels, resolution 1 pixel $\sim 0.05 \mathrm{~mm}$ ) were made to assess the proximal EDL tendon gap, which emerged when EDL contracted after proximal EDL tenotomy.

\subsection{Data analysis}

For each knee angle, ankle moments were calculated around three axes (axis perpendicular to the transverse plane: inversion $(+) /$ eversion; axis perpendicular to the sagittal plane: plantar-flexion/dorsi-flexion $(+)$; axis perpendicular to the frontal plane: external rotation $(+)$ /internal rotation) from forces and moments measured by the load cell using inverse static analysis (Tijs et al., 2014). Around each axis, ankle moments were assessed by calculating the mean over 50-ms time windows before (passive) and during individual TA and EDL excitation as well as during simultaneous TA and EDL (TA\&EDL) excitation. To obtain active TA, EDL and TA\&EDL ankle moments, the passive moment was subtracted from the ankle moment during muscle contraction. This assumes passive moments before and during muscle contraction to be equal, which is a reasonable approximation if
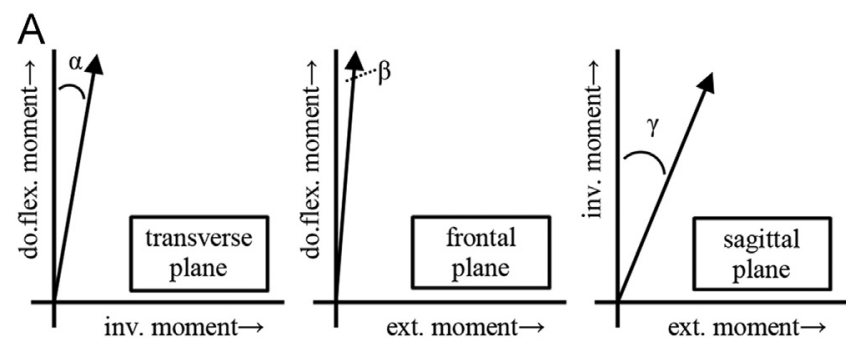

B

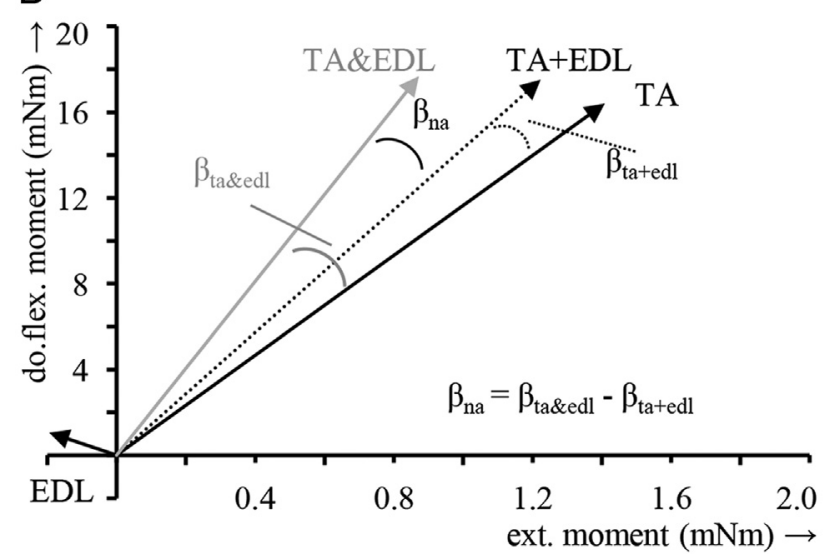

Fig. 2. Vector directions of exerted ankle moments. (A) For calculating TA vector direction in the transverse and frontal planes, the angle between the TA vector and the dorsi-flexion moment axis was calculated ( $\alpha$ and $\beta$, respectively). In the sagittal plane, the angle between the TA vector and the inversion moment axis $(\gamma)$ was calculated. Do.flex.: dorsi-flexion, inv.: inversion, ext.: external rotation. (B) Non-additive summation of vector directions in the frontal plane. Vector direction of individual TA and EDL ankle moments (black solid lines), of the summed ankle moment exerted on excitation of TA and EDL individually (TA+EDL, black dotted line), and of the ankle moment exerted on simultaneous excitation TA and EDL (TA\&EDL, gray solid line). For calculating non-additive direction summation in the frontal plane $\left(\beta_{\mathrm{na}}\right)$, the angle between TA and TA+EDL vectors $\left(\beta_{\mathrm{ta}+\mathrm{edl}}\right)$ was subtracted from the angle between TA and TA\&EDL $\left(\beta_{\text {ta\&edl }}\right)$. Positive value of $\beta_{\text {na }}$ indicates that the vector direction of TA\&EDL is directed more towards the moment direction of EDL than predicted based on the mathematical sum. Note that, for clarity, the ranges of both axes are not equal to each other. The data in this example were obtained with the ankle and knee angle kept at $90^{\circ}$ and $130^{\circ}$, respectively. passive forces are low (Rode et al., 2009). At $90^{\circ}$ ankle angle, both EDL (Huijing and Baan, 2008) and TA (Huijing and Baan, 2001a; Maas et al., 2001) are on the ascending limb of their length-force curves, indicating that the calculation of active moments is not substantially biased by this simplification.

TA, EDL and TA\&EDL ankle moments around each axis were used to calculate the magnitude of the $3 \mathrm{D}$ ankle moment vector and the direction of the projection of the 3D vector in the three anatomical planes. For TA vectors in the transverse and frontal planes, the angle relative to the dorsi-flexion moment axis ( $\alpha$ and $\beta$, respectively) was calculated. For TA vectors in the sagittal plane, the angle relative to the inversion moment axis $(\gamma)$ was calculated (Fig. 2A).

Non-additive magnitude summation was assessed by subtracting the mathematical sum of TA and EDL ankle moments (TA+EDL) from the TA\&EDL magnitude (Tijs et al., 2014). This was then normalized $\left(\% \mathrm{M}_{\text {na }}\right)$ relative to the magnitude of TA+EDL. Non-additive direction summation was assessed for each of the three anatomical planes (transverse plane: $\alpha_{\text {na }}$; frontal plane: $\beta_{\text {na }}$; sagittal plane: $\gamma_{\text {na }}$ ) by calculating the enclosed angle between the moment vector of TA+EDL and TA\&EDL (Fig. 2B for a frontal plane example).

Prior to and after each stage of dissection, control measurements were performed at a knee and ankle angle of $90^{\circ}$. Any changes in muscle conditions that could affect interpretation of the data were assessed.

\subsection{Statistics}

To test for effects of knee angle on TA ankle moment, one-way repeated measures ANOVAs (SPSS 20, IBM, USA) with 'knee angle' as independent factor were applied. Two-way repeated measures ANOVAs with 'knee angle' and 'dissection' as independent factors were applied to test for interaction effects between these factors on TA ankle moment. In addition, two levels of 'dissection' as independent factor ('intact' vs 'fasciotomy' and 'fasciotomy' vs 'tenotomy') were applied to perform planned comparisons for a main effect of dissection on TA moment. One-sample $t$-tests were used to test if non-additive summation averaged across knee angles was significantly different from zero. To test for effects of knee angle on non-additive summation of TA and EDL ankle moments, one-way repeated measures ANOVA with 'knee angle' as independent factor was applied. Paired t-tests were performed to assess effects of proximal EDL tenotomy on EDL ankle moment and to evaluate any changes in TA and EDL muscle conditions due to dissection and/or preceding muscle contractions. Greenhouse Geisser correction was used if the assumption of sphericity was violated. Level of significance was set at $p \leq 0.05$.

\section{Results}

\subsection{Effects of knee angle on 3D ankle moment of TA muscle}

Knee angle did not affect $(p=0.057)$ the magnitude of the 3D TA ankle moment (average across knee angles: $18.7 \pm 3.4 \mathrm{mNm}$, Fig. $3 \mathrm{~A}$ ).

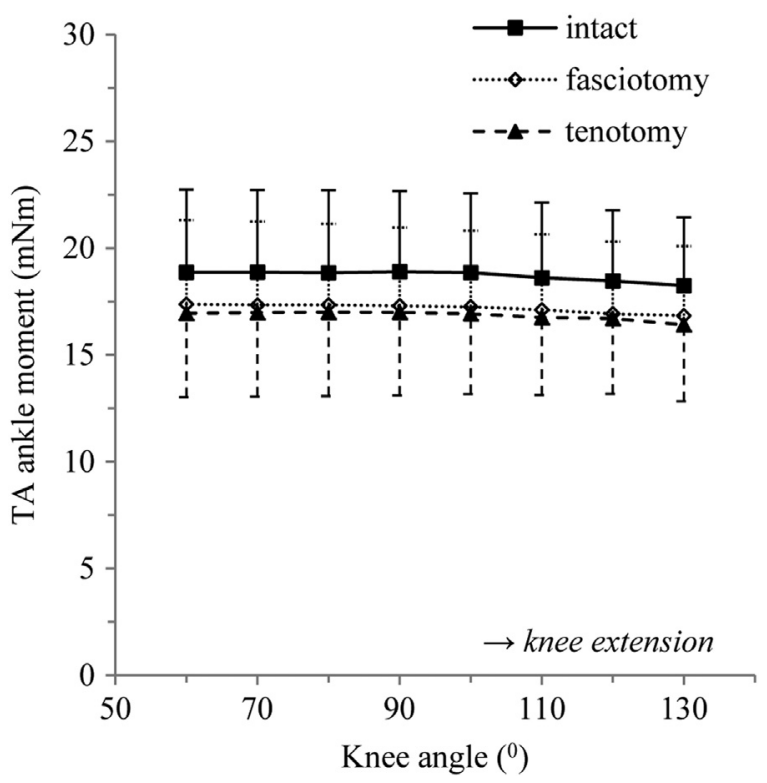

Fig. 3. Effects of knee angle on the magnitude of TA ankle moment. Effects of dissection (intact $\bullet$; fasciotomy $\diamond$; tenotomy $\mathbf{\wedge}$ ) on the magnitude of TA ankle moment plotted as a function of knee angle. Means \pm s.d. are shown $(n=8)$. 
A

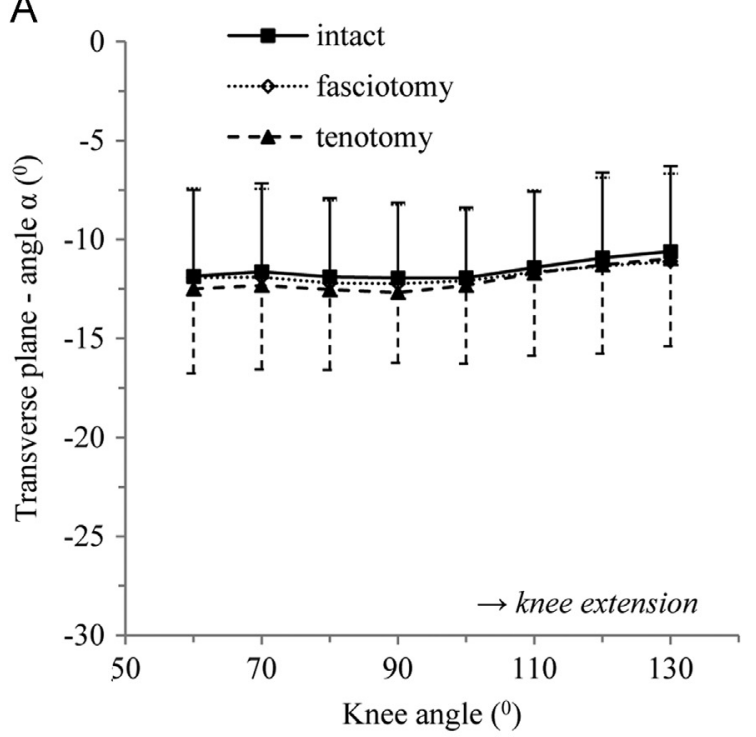

C

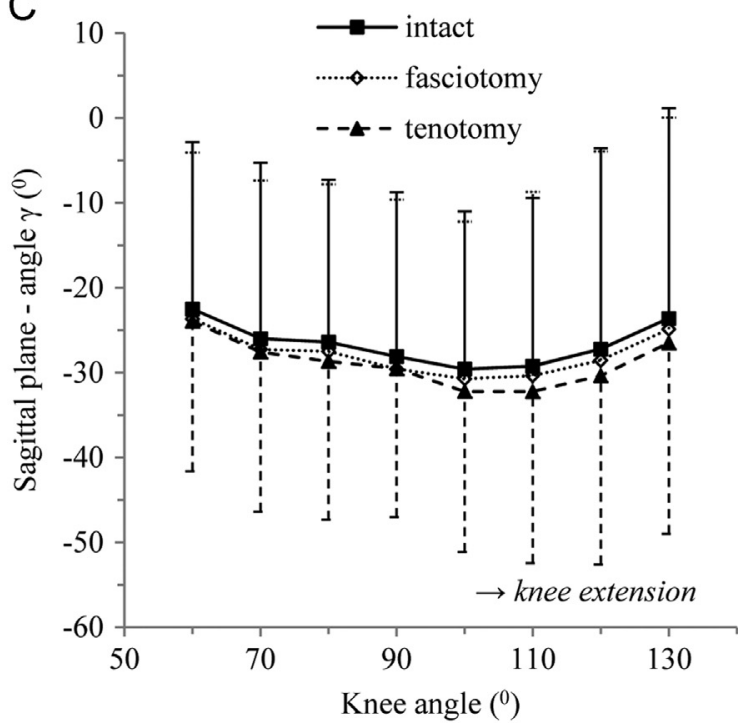

B

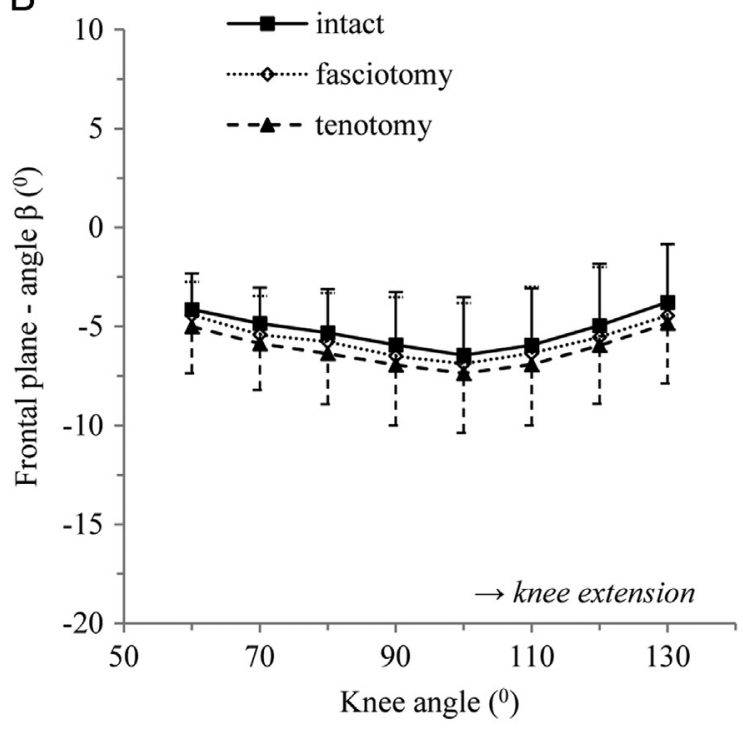

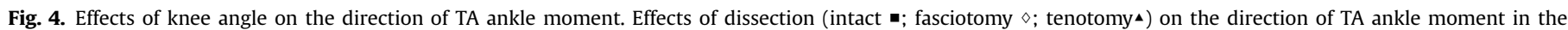
transverse (A), frontal (B) and sagittal (C) planes plotted as a function of knee angle. Means \pm s.d. are shown ( $n=8)$.

Although TA moment slightly decreased at extended knee angles (by $-0.6 \mathrm{mNm}$ ), control measurements indicated that this was not caused by fatigue or history effects $(\mathrm{p}=0.385)$. ANOVA indicated no interaction effect between dissection and knee angle $(\mathrm{p}=0.930)$, but fasciotomy decreased TA moment to an average of $17.2 \pm 3.4 \mathrm{mNm}$ $(p<0.001$, Fig. 3). Following proximal EDL tenotomy, TA moment decreased further to an average of $16.8 \pm 3.6 \mathrm{mNm}(p=0.037)$. This change, however, was not significant $(p=0.231)$ if corrected for the observed decrease in the control measurements $(-0.3 \pm 0.3 \mathrm{mNm}$, $p=0.023$ ).

Knee angle did affect TA moment vector direction, but only in the frontal plane $(\beta, p=0.035$, Fig. $4 \mathrm{~B})$. Extending the knee from $60^{\circ}$ to $100^{\circ}$ increased the direction from $\beta=-4.1 \pm 1.8^{\circ}$ to a peak angle of $\beta=-6.5 \pm 2.9^{\circ}$. Thereafter, the angle decreased to $\beta=-3.8 \pm 2.9^{\circ}$ at $130^{\circ}$. ANOVA indicated neither a main effect of dissection on vector direction (transverse plane: $p=0.094$, frontal plane: $p=0.147$, sagittal plane: $\mathrm{p}=0.304)$ nor interaction effects between dissection and knee angle (transverse plane: $p=0.763$, frontal plane: $p=0.994$, sagittal plane: $p=0.973$ ).

\subsection{Summation of TA and EDL ankle moments}

A small but significant ( $p=0.004)$ non-additive magnitude moment summation of $+1.1 \pm 1.1 \% \quad(+0.2 \pm 0.2 \mathrm{mNm}$, $p=0.003$ ) averaged across all knee angles was found (Fig. 5A). Knee angle did not affect the extent of non-additive summation ( $p=0.438)$. Non-additive summation of moment direction (Fig. 5B) was found only in the frontal plane $\left(\beta_{\mathrm{na}}, p=0.006\right)$ and was, in this case, knee angle dependent $(p<0.001)$. In that plane, non-additive direction summation ranged from $\beta_{\text {na }}=+0.2 \pm 0.3^{\circ}$ at $60^{\circ}$ to $\beta_{\text {na }}=+1.1 \pm 0.6^{\circ}$ at $130^{\circ}$. The positive summation angle indicates that the vector direction in the frontal plane during simultaneous excitation of TA and EDL is directed more towards the EDL vector than predicted based on the mathematical sum. In the transverse and sagittal planes, non-additive direction summation was not significantly different from zero $(p=0.203$ and $p=0.684$, respectively) and not knee angle dependent $(p=0.277$ and $p=0.594$, respectively). 

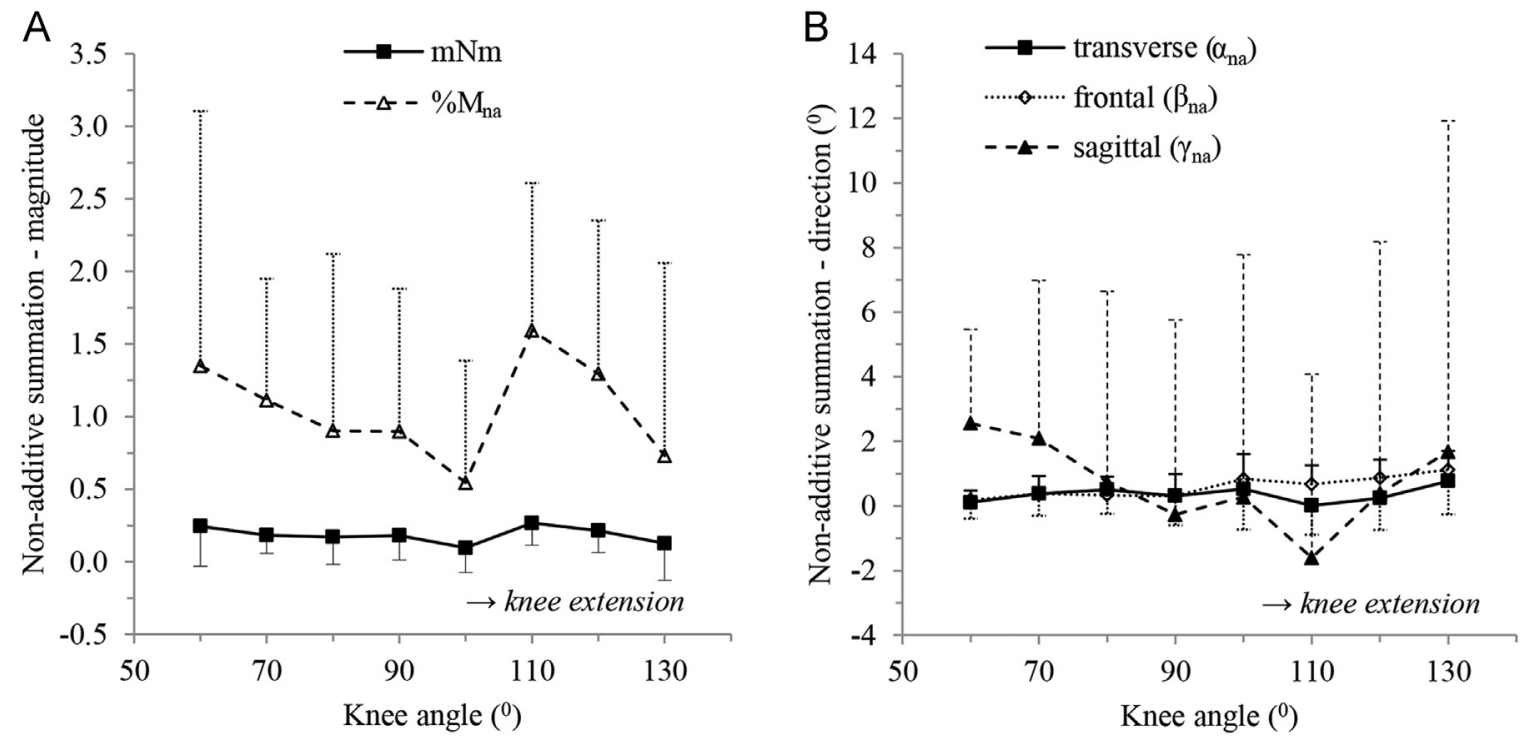

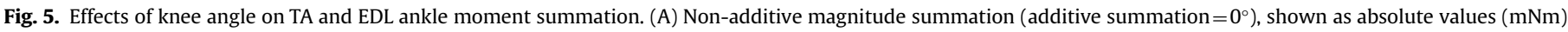

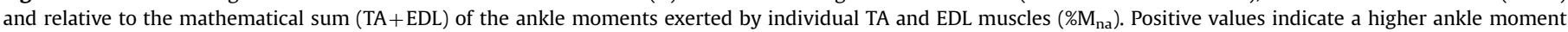

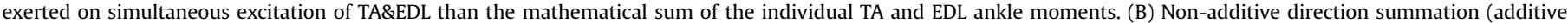
summation $\left.=0^{\circ}\right)$ in the transverse $\left(\alpha_{\text {na }}, \boldsymbol{\bullet}\right)$, frontal $\left(\beta_{\text {na }}, \diamond\right)$ and sagittal $\left(\gamma_{\text {na }}, \mathbf{\Delta}\right)$ planes plotted as a function of knee angle. Means \pm s.d. are shown $(n=8)$.

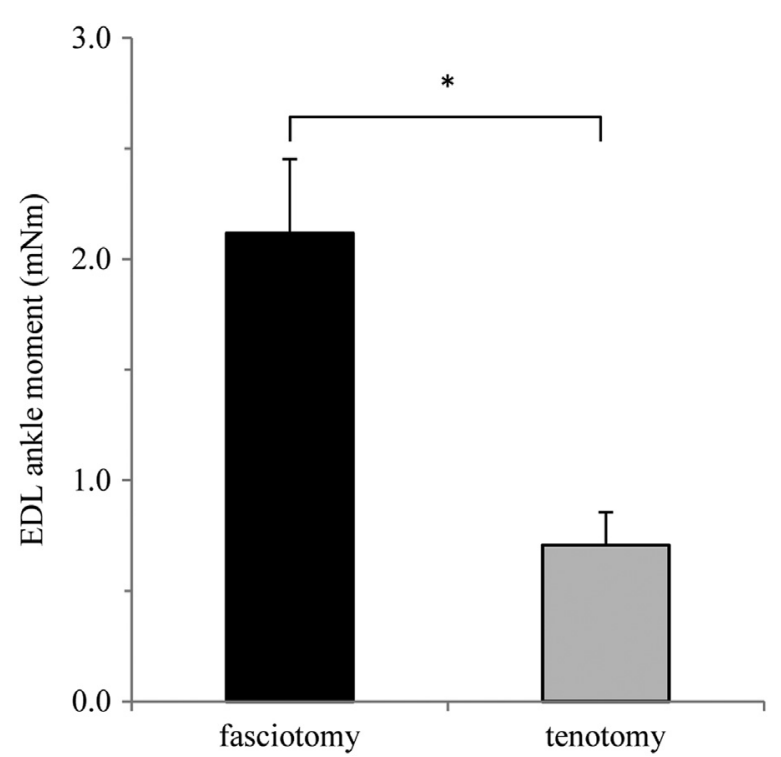

Fig. 6. Effects of proximal EDL tenotomy on EDL ankle moment. EDL ankle moment following fasciotomy (note that EDL could be excited only after fasciotomy, see Section 2) and after tenotomy are shown. Knee and ankle angle were kept at $90^{\circ}$. $* p<0.001$. Means \pm s.d. are shown $(n=8)$.

\subsection{Effects of proximal EDL tenotomy on the magnitude of EDL ankle moment}

Proximal EDL tenotomy decreased (Fig. 6, $p<0.001$ ) EDL ankle moment by $1.4 \pm 0.2 \mathrm{mNm}$ (by $67.1 \pm 4.3 \%$ ). After tenotomy, a tendon gap of $5.7 \pm 0.7 \mathrm{~mm}$ was found during contraction. This shortening of EDL muscle explains, at least partially, the decrease in ankle moment. Despite severing its origin, a substantial moment was still exerted on excitation of EDL $(0.7 \pm 0.1 \mathrm{mNm})$. This indicates that connective tissue linkages prevented EDL muscle fibers to shorten to active slack length.

\section{Discussion}

The main findings of the present study are that (1) TA ankle moment was not affected by changes in knee angle, which involves proximal length changes of passive EDL; (2) although summation of TA and EDL ankle moments was non-additive, the extent was only minimally affected by knee angle; and (3) EDL did exert a substantial moment after its origin was severed, which confirms the existence of strong myofascial connections between EDL and its surroundings.

\subsection{Effects of epimuscular myofascial connections on TA ankle moment}

The existence of epimuscular connective tissues between muscles in the anterior crural compartment of the rat hindlimb and their surrounding structures have been investigated extensively. Force exerted at the tied distal tendons of TA and extensor hallucis longus (EHL) increased when the position of EDL, kept at a constant MTU length, was moved from a distal to a proximal position relative to TA and EHL (Maas et al., 2004). Distal TA and EHL force decreased when EDL was lengthened distally (Huijing and Baan, 2003, 2008). These conditions were beyond physiological ranges considering that the MTU length of TA, EDL and EHL change to a similar extent during ankle joint movements. Changes in knee angle, however, result in EDL length changes exclusively and, hence, in relative muscle displacements. In contrast to distal length changes, lengthening EDL proximally was reported not to affect distal TA and EHL forces (Huijing and Baan, 2001a, 2003, 2008; Huijing et al., 2003). This is in agreement with the results of the present study, although we activated TA only instead of the whole synergistic muscle group. Therefore, the absence of coactivation can most likely not explain the lack of epimuscular myofascial force transmission (see next section on co-activation). These results suggest that intermuscular connections in the anterior crural compartment are organized in such a way that its net effect does not influence the mechanical function of neighboring muscles if the range of relative muscle displacements is within physiological limits. 
Even though fasciotomy did not affect the relationship between knee angle and TA ankle moment, it decreased the moment magnitude. This is in agreement with a decrease in muscle force following fasciotomy observed in dogs (Garfin et al., 1981) and rats (Huijing and Baan, 2001b; Smeulders et al., 2002). Such effects of fasciotomy have been attributed to extramuscular myofascial connections (Huijing and Baan, 2001b), or to a decrease in intramuscular pressure (Garfin et al., 1981). Fasciotomy could also have increased muscle bulging during muscle contraction, resulting in higher pennation angles (Hodges et al., 2003; Wakeling et al., 2013). In addition, muscle fibers can shorten more upon contraction after fasciotomy, as shortening is not restricted by connections with the fascia. This could decrease the force exerted at the distal TA tendon and, hence, reduce TA ankle moment. In the present study, however, we cannot distinguish between these mechanisms.

We found a small effect of knee angle on TA moment direction, however, this change was not caused by proximal EDL lengthening. Thus, other factors should be involved. Knee extension causes displacement of the sciatic nerve in proximal direction in humans (Ellis, 2012) and, hence, also of the common peroneal nerve. This may have resulted in changes of myofascial loads exerted by the neurovascular tract onto TA. In addition, tibiofemoral contact points may change as a function of knee angle due to the fact that the knee joint does not act as a perfect hinge (Hildebrand et al., 1991).

\subsection{Effects of epimuscular myofascial connections on TA and EDL ankle moment summation}

Co-activation of nearby muscles has been suggested to stiffen connective tissue linkages and, hence, facilitate epimuscular myofascial force transmission (Maas and Sandercock, 2010). To date, however, no evidence exists supporting this hypothesis (Maas and Huijing, 2009; Tijs et al., 2015a). In the present study, we did find that knee extension (i.e. proximal decrease in EDL MTU length) increased non-additive direction summation when TA and EDL were active. Although this suggests effects of coactivation on epimuscular myofascial force transmission, the increase was very small $\left(\sim 1^{\circ}\right)$. We also found very limited positive non-additive magnitude summation $(+1 \%)$. This could be explained by a more efficient transmission of force during coactivation (Sheard et al., 1999) via epimuscular myofascial pathways between TA and EDL muscle fibers. In addition, an increase in muscle moment arm (Akagi et al., 2012; Maganaris et al., 1999, 1998) or an increase in compartmental pressure due to coactivation (Garfin et al., 1981) could have increased muscle force and could also explain the positive non-additive summation. On the other hand, muscle bulging of neighboring muscles due to coactivation could have induced a transverse muscle load, which has been shown to reduce longitudinal muscle force (Siebert et al., 2014). However, this would have resulted in a negative nonadditive summation of TA and EDL ankle moments and can, therefore, not explain the positive non-additive summation found in the present study.

Although the various mechanisms cannot be distinguished in the present study, the net effect of intermuscular connections at the ankle joint were limited for physiological ranges of knee joint motion. This appears in contrast with recent imaging studies in humans that detected changes in fascicle length of the one-joint SO muscle if the gastrocnemius muscle is lengthened proximally via knee extension (Bojsen-Møller et al., 2010; Diong and Herbert, 2015; Tian et al., 2012). In addition, knee extension caused substantial values of local strain within SO (Huijing et al., 2011) and anterior crural muscles (Yaman et al., 2013). It is important to note that the one-joint muscle was active in the present study and passive in the imaging studies. Recently, we also found effects of epimuscular myofascial force transmission on the distribution of sarcomere lengths within a passive TA in rats (Tijs et al., 2015b), while no effects on the joint moment exerted by TA were found in the present study. This suggests that effects of epimuscular myofascial force transmission that occur in passive muscle conditions do not necessarily have the same impact in active muscle conditions.

\subsection{Functional implications}

The results of the present study are relevant for constructing biomechanical models and for functional electrical stimulation to control limb movements. Both applications assume muscles to be independent actuators. Recently, endpoint forces were assessed for several combinations of rat hindlimb muscles, and close to additive summations of muscle force vectors were found (Jarc et al., 2013). The results of the present study are in agreement with that study.

TA and EDL have opposite mechanical effects at the ankle joint (TA: inversion, EDL: eversion). The very limited net effects of epimuscular myofascial force transmission at the joint level allows these muscles to act independently. This may be advantageous for accurate control of ankle joint stability. This seems to be in contrast to a recent study showing that lengthening gastrocnemius and plantaris muscles proximally, simulating knee extension, increased the force exerted at the distal tendon of soleus muscle significantly (Bernabei et al., 2015). However, because ankle plantar-flexors have largely comparable lines of action (Tijs et al., 2014), the presence of myofascial force transmission will not affect the functional output at the joint. Recent studies (Maas and Sandercock, 2008; Tijs et al., 2015a) did indeed found no effects of knee extension on soleus ankle moment. Nonetheless, force transmission between triceps surae muscles may serve as a mechanism to reduce local stresses by distributing stresses and strains over multiple muscles and tendons (Bojsen-Møller et al., 2010).

\section{Conclusion}

We found very limited effects of proximal EDL length changes on the mechanical output effect of TA at the ankle joint. Therefore, we conclude that myofascial connections between muscles in the anterior crural compartment have limited mechanical relevance if synergistic length changes are within ranges that occur during normal movements.

\section{Conflict of interest statement}

No competing interests declared.

\section{Acknowledgment}

The authors would like to thank Guus Baan for designing a schematic 3D model of the branches of the common peroneal nerve. This study was supported by the Division for Earth and Life Sciences of the Netherlands Organization for Scientific Research [864-10-011]. The funding agency had no involvement in the study design; in the collection, analysis and interpretation of data; in writing the manuscript; and in the decision to submit the manuscript for publication. 


\section{References}

Akagi, R., Iwanuma, S., Hashizume, S., Kanehisa, H., Yanai, T., Kawakami, Y., 2012 In vivo measurements of moment arm lengths of three elbow flexors at rest and during isometric contractions. J. Appl. Biomech. 28, 63-69.

Bauman, J.M., Chang, Y.H., 2010. High-speed X-ray video demonstrates significant skin movement errors with standard optical kinematics during rat locomotion. J. Neurosci. Methods 186, 18-24. http://dx.doi.org/10.1016/j. jneumeth.2009.10.017.

Bernabei, M., van Dieën, J.H., Baan, G.C., Maas, H., 2015. Significant mechanical interactions at physiological lengths and relative positions of rat plantar flexors. J. Appl. Physiol. 118, 427-436. http://dx.doi.org/10.1152/ japplphysiol.00703.2014.

Bojsen-Møller, J., Schwartz, S., Kalliokoski, K.K., Finni, T., Magnusson, S.P., 2010. Intermuscular force transmission between human plantarflexor muscles in vivo. J. Appl. Physiol. 109, 1608-1618. http://dx.doi.org/10.1152 japplphysiol.01381.2009.

Diong, J., Herbert, R.D., 2015. Is ankle contracture after stroke due to abnormal intermuscular force transmission? J. Appl. Biomech. 31, 13-18. http://dx.doi. org/10.1123/jab.2014-0064.

Ellis, R.F., 2012. Comparison of different neural mobilization exercises upon longitudinal sciatic nerve movement: an in-vivo study utilizing ultrasound imaging J. Orthop. Sport. Phys. Ther. 42, 667-675. http://dx.doi.org/10.2519/ jospt.2012.3854.

Garfin, S.R., Tipton, C.M., Mubarak, S.J., Woo, S.L., Hargens, A.R., Akeson, W.H., 1981 Role of fascia in maintenance of muscle tension and pressure. J. Appl. Physiol $51,317-320$

Greene, E.C., 1935. Anatomy of the rat. Trans. Am. Philos. Soc.

Gruner, J.A., Altman, J., 1980. Swimming in the rat: analysis of locomotor performance in comparison to stepping. Exp. Brain Res. 40, 374-382. http://dx.doi. org/10.1007/BF00236146.

Gruner, J.A., Altman, J., Spivack, N., 1980. Effects of arrested cerebellar development on locomotion in the rat - Cinematographic and electromyographic analysis. Exp. Brain Res. 40, 361-373. http://dx.doi.org/10.1007/BF00236145.

Hildebrand, C., Oqvist, G., Brax, L., Tuisku, F., 1991. Anatomy of the rat knee joint and fibre composition of a major articular nerve. Anat. Rec. 229, 545-555. http://dx.doi.org/10.1002/ar.1092290415.

Hodges, P.W., Pengel, L.H.M., Herbert, R.D., Gandevia, S.C., 2003. Measurement of muscle contraction with ultrasound imaging. Muscle Nerve 27, 682-692. http://dx.doi.org/10.1002/mus.10375.

Huijing, P.A., 2009. Epimuscular myofascial force transmission: A historical review and implications for new research. International society of biomechanics Muybridge award lecture, Taipei, 2007. J. Biomech. 42, 9-21. http://dx.doi.org 10.1016/j.jbiomech.2008.09.027.

Huijing, P.A., Baan, G.C., 2001a. Extramuscular myofascial force transmission within the rat anterior tibial compartment: proximo-distal differences in muscle force. Acta Physiol. Scand. 173, 297-311. http://dx.doi.org/10.1046/j.1365-201X.2001.00911.x.

Huijing, P.A. Baan, G.C., 2001b. Myofascial force transmission causes interaction between adjacent muscles and connective tissue: effects of blunt dissection and compartmental fasciotomy on length force characteristics of rat extenso digitorum longus muscle. Arch. Physiol. Biochem. 109, 97-109. http://dx.doi org/10.1076/apab.109.2.97.4269.

Huijing, P.A., Baan, G.C., 2003. Myofascial force transmission: muscle relative position and length determine agonist and synergist muscle force. J. Appl Physiol. 94, 1092-1107. http://dx.doi.org/10.1152/japplphysiol.00173.2002.

Huijing, P.A., Baan, G.C., 2008. Myofascial force transmission via extramuscular pathways occurs between antagonistic muscles. Cells Tissues Organs 188 400-414. http://dx.doi.org/10.1159/000118097.

Huijing, P.A., Maas, H., Baan, G.C., 2003. Compartmental fasciotomy and isolating a muscle from neighboring muscles interfere with myofascial force transmission within the rat anterior crural compartment. J. Morphol. 256, 306-321. http://dx.doi.org/10.1002/jmor.10097.

Huijing, P.A., van de Langenberg, R.W., Meesters, J.J., Baan, G.C., 2007. Extramuscular myofascial force transmission also occurs between synergistic muscles and antagonistic muscles. J. Electromyogr. Kinesiol. 17, 680-689. http://dx.doi.org/ 10.1016/j.jelekin.2007.02.005.

Huijing, P.A., Yaman, A., Ozturk, C., Yucesoy, C.A., 2011. Effects of knee joint angle on global and local strains within human triceps surae muscle: MRI analysis indicating in vivo myofascial force transmission between synergistic muscles. Surg. Radiol. Anat. 33, 869-879. http://dx.doi.org/10.1007/s00276-011-0863-1.

Jarc, A.M., Berniker, M., Tresch, M.C., 2013. FES control of isometric forces in the rat hindlimb using many muscles. IEEE Trans. Biomed. Eng. 60, 1422-1430. http://dx.doi.org/10.1109/TBME.2013.2237768.

Johnson, W.L., Jindrich, D.L., Roy, R.R., Edgerton, V.R., 2008. A three-dimensional model of the rat hindlimb: Musculoskeletal geometry and muscle moment arms. J. Biomech. 41, 610-619. http://dx.doi.org/10.1016/j.jbiomech.2007.10.004.
Kawakami, Y., Ichinose, Y., Fukunaga, T., 1998. Architectural and functional features of human triceps surae muscles during contraction. J. Appl. Physiol. 85, 398-404.

Maas, H., Sandercock, T.G., 2008. Are skeletal muscles independent actuators? Force transmission from soleus muscle in the cat. J. Appl. Physiol. 104, 1557-1567. http://dx.doi.org/10.1152/japplphysiol.01208.2007.

Maas, H., Huijing, P.A., 2009. Synergistic and antagonistic interactions in the rat forelimb: acute effects of coactivation. J. Appl. Physiol. 107, 1453-1462. http://dx.doi.org/10.1152/japplphysiol.00328.2009.

Maas, H., Sandercock, T.G., 2010. Force transmission between synergistic skeletal muscles through connective tissue linkages. J. Biomed. Biotechnol. 2010, 575672. http://dx.doi.org/10.1155/2010/575672.

Maas, H., Baan, G.C. Huijing, P.A., 2001. Intermuscular interaction via myofascial force transmission: effects of tibialis anterior and extensor hallucis longus length on force transmission from rat extensor digitorum longus muscle. J. Biomech. 34, 927-940. http://dx.doi.org/10.1016/S0021-9290(01)00055-0.

Maas, H., Baan, G.C., Huijing, P.A., 2004. Muscle force is determined also by muscle relative position: isolated effects. J. Biomech. 37, 99-110. http://dx.doi.org/ 10.1016/S0021-9290(03)00235-5.

Maas, H., Meijer, H.J.M., Huijing, P.A., 2005. Intermuscular interaction between synergists in rat originates from both intermuscular and extramuscular myofascial force transmission. Cells Tissues Organs 181, 38-50. http://dx.doi.org/ $10.1159 / 000089967$.

Maganaris, C.N., Baltzopoulos, V., Sargeant, A.J., 1998. Changes in Achilles tendon moment arm from rest to maximum isometric plantarflexion: in vivo observations in man. J. Physiol. 510, 977-985.

Maganaris, C.N., Baltzopoulos, V., Sargeant, A.J., 1999. Changes in the tibialis anterior tendon moment arm from rest to maximum isometric dorsiflexion: in vivo observations in man. Clin. Biomech. 14, 661-666. http://dx.doi.org/10.1016/ S0268-0033(99)00018-2.

Meijer, H.J.M., Baan, G.C., Huijing, P.A., 2006. Myofascial force transmission is increasingly important at lower forces: firing frequency-related length-force characteristics of rat extensor digitorum longus. Acta Physiol. 186, 185-195. http://dx.doi.org/10.1111/j.1748-1716.2006.01528.x.

Rijkelijkhuizen, J.M., Meijer, H.J.M., Baan, G.C., Huijing, P.A., 2007. Myofascial force transmission also occurs between antagonistic muscles located within opposite compartments of the rat lower hind limb. J. Electromyogr. Kinesiol. 17, 690-697. http://dx.doi.org/10.1016/j.jelekin.2007.02.004.

Rode, C., Siebert, T., Herzog, W., Blickhan, R., 2009. The effects of parallel and series elastic components on the active cat soleus force-length relationship. J. Mech. Med. Biol. 9, 105-122. http://dx.doi.org/10.1142/S0219519409002870.

Schmidt, A., Fischer, M.S., 2011. The kinematic consequences of locomotion on sloped arboreal substrates in a generalized (Rattus norvegicus) and a specialized (Sciurus vulgaris) rodent. J. Exp. Biol. 214, 2544-2559. http://dx.doi.org/ 10.1242 jeb.051086.

Sheard, P.W., Mchannigan, P., Duxson, M.J., 1999. Single and paired motor unit performance in skeletal muscles: comparison between simple and series-fibred muscles from the rat and the guinea pig. Basic Appl. Myol. 9, 79-87.

Siebert, T., Till, O., Blickhan, R., 2014. Work partitioning of transversally loaded muscle: experimentation and simulation. Comput. Methods Biomech. Biomed. Eng. 17, 217-229. http://dx.doi.org/10.1080/10255842.2012.675056.

Smeulders, M.J.C., Kreulen, M., Joris Hage, J., Baan, G.C., Huijing, P.A., 2002. Progressive surgical dissection for tendon transposition affects length-force characteristics of rat flexor carpi ulnaris muscle. J. Orthop. Res. 20, 863-868. http://dx.doi.org/10.1016/S0736-0266(01)00181-4.

Tian, M., Herbert, R.D., Hoang, P., Gandevia, S.C., Bilston, L.E., 2012. Myofascial force transmission between the human soleus and gastrocnemius muscles during passive knee motion. J. Appl. Physiol. 113, 517-523. http://dx.doi.org/10.1152/ japplphysiol.00111.2012.

Tijs, C., van Dieën, J.H., Maas, H., 2015a. No functionally relevant mechanical effects of epimuscular myofascial connections between rat ankle plantar flexors. J. Exp. Biol. 218, 2935-2941. http://dx.doi.org/10.1242/jeb.122747.

Tijs, C., van Dieën, J.H., Maas, H., 2015b. Effects of epimuscular myofascial force transmission on sarcomere length of passive muscles in the rat hindlimb. Physiol. Rep. 3, e12608. http://dx.doi.org/10.14814/phy2.12608.

Tijs, C., van Dieën, J.H., Baan, G.C., Maas, H., 2014. Three-dimensional ankle moments and nonlinear summation of rat triceps surae muscles. PLoS One 9, e111595. http://dx.doi.org/10.1371/journal.pone.0111595.

Wakeling, J.M., Jackman, M., Namburete, A.I., 2013. The effect of external compression on the mechanics of muscle contraction. J. Appl. Biomech. 29, 360-364.

Yaman, A., Ozturk, C., Huijing, P.A., Yucesoy, C.A., 2013. Magnetic resonance imaging assessment of mechanical interactions between human lower leg muscles in vivo. J. Biomech. Eng. 135, 91003. http://dx.doi.org/10.1115/1.4024573. 